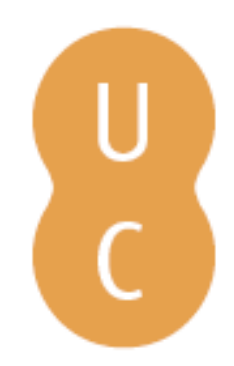

\title{
pompalina
}

\section{La mandrágora de Amato Lusitano: edición, traducción y anotación}

Autor(es): González Manjarrés, Miguel Ángel

Publicado por: UA Editora - Universidade de Aveiro; Imprensa da Universidade de

URL

persistente: URI:http://hdl.handle.net/10316.2/35705

DOI: $\quad$ DOI:http://dx.doi.org/10.14195/978-989-26-0941-6_19

Accessed : $\quad$ 26-Apr-2023 15:59:10

A navegação consulta e descarregamento dos títulos inseridos nas Bibliotecas Digitais UC Digitalis, UC Pombalina e UC Impactum, pressupõem a aceitação plena e sem reservas dos Termos e Condições de Uso destas Bibliotecas Digitais, disponíveis em https://digitalis.uc.pt/pt-pt/termos.

Conforme exposto nos referidos Termos e Condições de Uso, o descarregamento de títulos de acesso restrito requer uma licença válida de autorização devendo o utilizador aceder ao(s) documento(s) a partir de um endereço de IP da instituição detentora da supramencionada licença.

Ao utilizador é apenas permitido o descarregamento para uso pessoal, pelo que o emprego do(s) título(s) descarregado(s) para outro fim, designadamente comercial, carece de autorização do respetivo autor ou editor da obra.

Na medida em que todas as obras da UC Digitalis se encontram protegidas pelo Código do Direito de Autor e Direitos Conexos e demais legislação aplicável, toda a cópia, parcial ou total, deste documento, nos casos em que é legalmente admitida, deverá conter ou fazer-se acompanhar por este aviso.

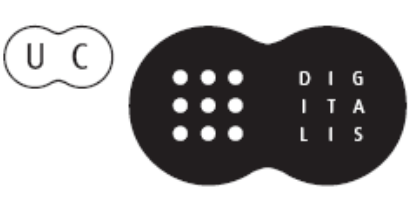




\section{HUMANISMO E CIÊNCIA Antiguidade e Renascimento}

António Manuel Lopes Andrade

Carlos de Miguel Mora

João Manuel Nunes Torrão
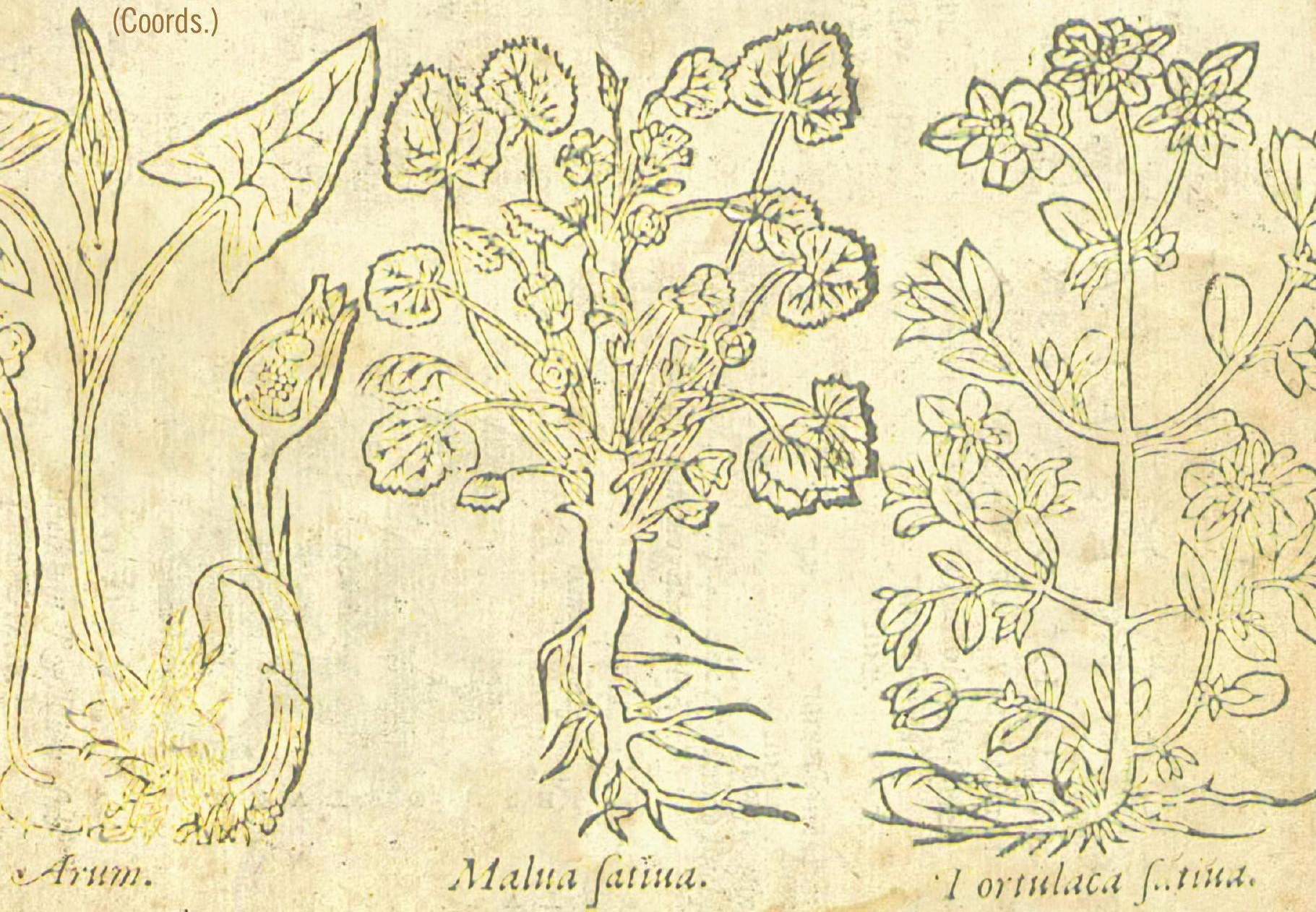

Aveiro I Coimbra I São Paulo 2015

UA Editora - Universidade de Aveiro I Imprensa da Universidade de Coimbra I Annablume 
Este volume resulta de várias iniciativas desenvolvidas no âmbito do projecto de I\&D "Dioscórides e o Humanismo Português: os Comentários de Amato Lusitano" (http://amatolusitano.web.ua.pt), recoIhendo contribuições de mais de duas dezenas de colaboradores, tanto de membros da equipa como de outros investigadores nacionais e estrangeiros. Entre os eventos que estiveram na origem deste livro destacam-se as três edições do Ciclo de Conferências promovido pelo projecto, realizadas entre 2010 e 2013, e sobretudo o Colóquio Internacional "Dioscórides e o Humanismo Português: os Comentários de Amato Lusitano", que decorreu no Departamento de Línguas e Culturas da Universidade de Aveiro, nos dias 21 e 22 de Novembro de 2013.

0 objectivo principal do projecto é a edição e tradução para português dos dois livros que Amato Lusitano dedicou ao comentário do tratado grego De materia medica de Dioscórides, ou seja, o Index Dioscoridis (Antuérpia, 1536) e as In Dioscoridlis Anazarbei de medica materia libros quinque... enarrationes (Veneza, 1553), estando contemplada, também, a tradução de mais duas obras directamente correlacionadas com os livros do médico português: a montante, a do próprio tratado grego de Dioscórides; a jusante, a do livro intitulado Apologia adversus Amathum Lusitanum (Veneza, 1558) de Pietro Andrea Mattioli.

OBRA PUBLICADA COM A COORDENAÇÃO

CIENTÍFICA DE:

Centro de Línguas, Literaturas e Culturas da Universidade de Aveiro

Centro de Estudos Clássicos e Humanísticos da Universidade de Coimbra

Cátedra de Estudos Sefarditas "Alberto

Benveniste" da Faculdade de Letras da Universidade de Lisboa 


\section{HUMANISMO E CIÊNCIA}

\section{Antiguidade e Renascimento}

ANTÓNIO MANUEL LOPES ANDRADE

CARLOS DE MIGUEL MORA

JOÃO MANUEL NUNES TORRÃO

(COORDS.)

AVEIRO • COIMBRA • SÃO PAULO

2015

UA EDITORA • UNIVERSIDADE DE AVEIRO

IMPRENSA DA UNIVERSIDADE DE COIMBRA

ANNABLUME 


\section{HUMANISMO E CIÊNCIA: Antiguidade e Renascimento}

\author{
EDIÇÃO

UA EDITORA • UNIVERSIDADE DE AVEIRO
IMPRENSA DA UNIVERSIDADE DE COIMBRA
ANNABLUME

ORGANIZAÇÃO E COORDENAÇÃO EDITORIAL ANTÓNIO MANUEL LOPES ANDRADE

CARLOS DE MIGUEL MORA

JOÃO MANUEL NUNES TORRÃO

\author{
DESIGN DA CAPA \\ MEIOKILO DESIGN STUDIO
}

DESIGN

CARLOS COSTA

IMPRESSÃO E ACABAMENTO

SERSILITO • MAIA

ISBN

UA • 978-972-789-434-5

IUC • 978-989-26-0940-9

\section{ISBN DIGITAL}

UA • 978-972-789-435-2

IUC • 978-989-26-0941-6

DOI

http://dx.doi.org/10.14195/ 978-989-26-0941-6

DEPÓSITO LEGAL 368241/13

TIRAGEM 500 Exemplares

(C) 2015

UA EDITORA • UNIVERSIDADE DE AVEIRO IMPRENSA DA UNIVERSIDADE DE COIMBRA

ANNABLUME

\section{COMISSÃO CIENTÍFICA}

António Manuel Lopes Andrade

Carlos de Miguel Mora

Delfim Ferreira Leão

Henrique Leitão

João Manuel Nunes Torrão

Maria de Fátima Reis

Maria do Céu Zambujo Fialho

Miguel Ángel González Manjarrés

\section{TEXTOS}

Adelino Cardoso

Ana Leonor Pereira

Ana Margarida Borges

António Guimarães Pinto

António Maria Martins Melo

Bernardo Mota

Carlos A. Martins de Jesus

Carlos de Miguel Mora

Cristina Santos Pinheiro

Donald Beecher

Emília Oliveira

Isabel Malaquias

James W. Nelson Novoa

Joana Mestre Costa

João Manuel Nunes Torrão

João Rui Pita

Jorge Paiva

José Sílvio Moreira Fernandes

Maria de Fátima Silva

Miguel Ángel González Manjarrés

Rui Manuel Loureiro

Telmo Corujo dos Reis

Teresa Nobre de Carvalho

Vinicije B. Lupis

Virgínia Soares Pereira 


\section{HUMANISMO E CIÊNCIA}

\section{Antiguidade e Renascimento}

ANTÓNIO MANUEL LOPES ANDRADE

CARLOS DE MIGUEL MORA

JOÃO MANUEL NUNES TORRÃO

(COORDS.)

AVEIRO • COIMBRA • SÃO PAULO

2015

UA EDITORA • UNIVERSIDADE DE AVEIRO

IMPRENSA DA UNIVERSIDADE DE COIMBRA

ANNABLUME 
OBRA PUBLICADA

COM A COORDENAÇÃO

CIENTÍFICA DE:

CENTRO DE LÍNGUAS,

LITERATURAS E CULTURAS DA

UNIVERSIDADE DE AVEIRO

CENTRO DE ESTUDOS

CLÁSSICOS E HUMANÍSTICOS DA

UNIVERSIDADE DE COIMBRA

CÁTEDRA DE ESTUDOS SEFARDITAS

"ALBERTO BENVENISTE"

DA FACULDADE DE LETRAS DA

UNIVERSIDADE DE LISBOA 


\section{SUMÁRIO}

PREFÁCIO

1.1 "Teofrasto, Tratado das plantas. No alvor de uma nova ciência" 13

Maria de Fátima Silva

1.2 "Francisco de Melo e os fragmentos de teoria óptica de Pierre Brissot" 21 Bernardo Mota

1.3 "Algumas reflexões sobre as pedras preciosas nos Colóquios dos simples de Garcia de Orta" 37 Rui Manuel Loureiro

1.4 "Estratégias, patronos e favores em Colóquios dos Simples de Garcia de Orta" 63 Teresa Nobre de Carvalho

1.5 "As plantas na obra poética de Camões (épica e lírica)" 95 Jorge Paiva

1.6 "Nicolás Monardes, John Frampton and the Medical Wonders of the New World" .141 Donald Beecher

1.7 "Literatura e Medicina: alguns textos de Justo Lípsio e de dois doutores Luís Nunes" 161 António Guimarães Pinto

1.8 "Ontologias e idiossincrasias dos Amantes, à luz da Archipathologia de Filipe Montalto" ...... 211 Joana Mestre Costa \& Adelino Cardoso

1.9 "Gabriel da Fonseca. A New Christian doctor in Bernini's Rome". .227 James W. Nelson Novoa 


\section{2) DIOSCÓRIDES E O HUMANISMO PORTUGUÊS: OS COMENTÁRIOS DE AMATO LUSITANO}

2.1 "Léxico científico português nos Comentários de Amato: antecedentes e receção"

Ana Margarida Borges

2.2 "Usos medicinais das plantas, em Amato Lusitano: o bálsamo"

António Maria Martins Melo

2.3 "Amato Lusitano e a importância da ilustração botânica no século xVI.

Em torno das edições lionesas das Enarrationes (1558)"

Carlos A. Martins de Jesus

2.4 "Sobre la identificación entre ébano y guayaco en una entrada

del Index Dioscoridis de Amato Lusitano".

Carlos de Miguel Mora

2.5 "Os partos distócicos em Amato Lusitano e em Rodrigo de Castro:

fontes, doutrinas e terapias greco-romanas"

\section{Cristina Santos Pinheiro}

2.6 "Do carvalho ao castanheiro: usos e propriedades medicinais

de fagáceas nas Enarrationes de Amato Lusitano".

Emília Oliveira

2.7 "O mundo mineral nos Comentários a Dioscórides de Amato Lusitano".

Isabel Malaquias \& Virgínia Soares Pereira

2.8 "Alguns comentários de Amato: entre a estranheza e a realidade"

João Manuel Nunes Torrão

2.9 "Caracterização e usos terapêuticos de produtos de origem marinha

nos Comentários de Amato Lusitano a Dioscórides"

José Sílvio Moreira Fernandes

2.10 "La mandrágora de Amato Lusitano: edición, traducción y anotación"

Miguel Ángel González Manjarrés

2.11 "O vinho e os vinhos - usos e virtudes de um dom dos deuses

nas Enarrationes de Amato Lusitano"

Telmo Corujo dos Reis

2.12 "Amatus Lusitanus e Didaco Pirro: due ebrei portoghesi

e cerchia umanistica di Dubrovnik" 481

Vinicije B. Lupis

2.13 "Estudos contemporâneos sobre Amato Lusitano". 513

João Rui Pita \& Ana Leonor Pereira 


\section{La mandrágora de Amato Lusitano: edición, traducción y anotación}

MIGUEL ÁNGEL GONZÁLEZ MANJARRÉS ${ }^{1}$

\section{RESUMEN:}

Se presenta una edición latina y traducción castellana anotada del capítulo de la mandrágora que incluyó Amato Lusitano (1511-1568) en sus Enarrationes (Venecia 1553) a Dioscórides. Se trata de plantear un posible modelo de trabajo para la edición y traducción de la obra completa, tal como se presentó en su día dentro del Proyecto de Investigación "Dioscórides e o humanismo português: os Comentários de Amato Lusitano".

PALABRAS CLAVE:

Amato Lusitano; mandrágora; edición; traducción.

\section{ABSTRACT:}

The chapter about mandrake in Amatus Lusitanus's Enarrationes (Venice 1553) is here edited in Latin and translated to Spanish. The text is also annotated. The article is a case-study for editing and translating the Amatus's whole comments on Dioscorides, and it was already presented at a workshop of the research project "Dioscorides and the Portuguese humanism: the Amatus Lusitanus's Comments".

\section{KEYWORDS:}

Amatus Lusitanus; mandrake; edition; translation.

1 Universidad de Valladolid: miguelan@fyl.uva.es. 


\section{INTRODUCCIÓN}

\section{PRELIMINAR}

El texto que aquí se presenta tuvo su origen en una suerte de "case-study" planteado en una de las reuniones de trabajo del Equipo de Investigación que ha llevado a cabo el Proyecto "Dioscórides e o humanismo português: os Comentários de Amato Lusitano". Se trataba entonces de plantear y discutir una posible metodología para afrontar la edición, traducción y anotación de las Enarrationes a Dioscórides del médico portugués Amato Lusitano (1511-1568) y, a partir de un ejemplo concreto, afrontar los problemas principales que pudieran surgir. Se eligió para ello el caso de la mandrágora, una planta célebre desde antiguo que contó con una tradición paralela - aunque a veces también concordante — en el ámbito de la medicina popular y de la medicina docta, y a cuyo estudio nos habíamos acercado ya desde otros puntos de vista, lo que ofrecía un terreno más firme para el propósito perseguido. Fruto de aquel trabajo, por tanto, es esta edición latina y traducción castellana del comentario de Amato, que pasamos a contextualizar brevemente para su mejor comprensión.

\section{LA MANDRÁGORA DE DIOSCÓRIDES}

Desde tiempos muy antiguos se usó la mandrágora como remedio farmacéutico. Debido a la figuración antropomórfica que se adivinó en su rizoma y a sus propios componentes alcalinos, la mandrágora se vio envuelta desde pronto en leyendas, fábulas y ceremonias relacionadas con la magia y la hechicería, que tuvieron una plasmación práctica en la medicina popular y que, poco a poco, fueron aumentando en la Edad Media y el Renacimiento hasta casi constituir hoy día la cualidad misma de la planta ${ }^{2}$.

2 Para la historia de la mandrágora baste citar los siguientes estudios de conjunto: Charles J. S. THOMSON, The Mystic Mandrake. New York, University Books, 1968; Jean-Denis RoLAND, "La mandragore: le mythe d'une racine, la racine d'un mythe", Annales des Sciences Naturells, Botanique 2 (1990-1991), pp. 49-81; Dorit Wittuın, Mandragora. Eine Arzneipflanze in Antike, Mittelalter und Neuzeit. Basel, Dietikon, 1999; Frederik J. Simoons, Plants of Life, Plants of Death. Madison-London, The University of Wisconsin Press, 1999, pp. 101-135; Vera HAMBEL, Die alte Heydnische Abgöttische Fabel von der Alraun: Verwendung und Bedeutung der Alraune in Geschichte und Gegenwart. Diss., Passau, Universität Passau, 2002; Thierry ZARCONE, "Le Mythe de la mandragore: la plante-homme", Diogenes 207 (2004), pp. 140-173; Anne van ArSdall-Helmut W. Klug-Paul Blanz, "The Mandrake Plant and its Legend: A New Perspective", in Peter Bierbaumer-Helmut W. Klug (eds.), Old Names-New Growth: Proceedings of the 2nd ASPNS Conference. Graz, Universität Graz, 2007, pp. 285-346; Marlene VAN DEN BERG-Marianne DIRCKSEN, "Mandrake from Antiquity to Harry Potter", Akroterion 53 (2008), pp. 67-79. 
La tradición popular insistió en su condición humanoide, cuyo origen se explicaría en leyendas muy antiguas: la planta habría brotado del semen que el primer hombre derramó sobre la tierra. En relación con tan curioso nacimiento deberían situarse las dos cualidades que han hecho más célebre a la mandrágora: su valor erótico y su actividad para volver fértiles a mujeres estériles, como se aprecia ya en los textos bíblicos ${ }^{3}$. De hecho, la medicina popular siguió empleando la planta en tales usos y, según frecuentes denuncias de farmacólogos renacentistas, los vendedores ambulantes de boticas y bebedizos la falsificaban con otras semejantes y la vendían a mujeres con problemas para engendrar ${ }^{4}$.

Pero hubo también leyendas relacionadas con su recolección ${ }^{5}$. Teofrasto, por ejemplo, cuenta que debían realizarse tres círculos a su alrededor con una espada y recolectarla mirando a Occidente, al tiempo que, al cortar un segundo trozo, era preciso bailar y decir numerosas retahílas de asunto erótico ${ }^{6}$. La ceremonia, no obstante, se fue complicando con el tiempo: en la Guerra contra los judios de Flavio Josefo se cuenta cómo la planta tenía casi sentimientos, huía de quien se acercaba a ella (solo quedaba paralizada si se le echaba encima orina de mujer o sangre menstrual) y, cuando la arrancaban, el recolector debía atar un perro a su raíz para que tirase de ella, pues quien la sacaba de tierra moría al instante ${ }^{7}$.

En cualquier caso, y de forma paralela a su amplia difusión popular y literaria, el uso racional y "científico" de la mandrágora entró también en la literatura farmacológica y médica (Corpus Hippocraticum, Teofrasto, Dioscórides, Plinio, Galeno, etc.), en que fue objeto de

3 Véase F. J. Simoons, Plants of Life..., op. cit., pp. 107-108; V. HAMBEL, Die alte Heydnische..., op. cit., pp. 27-30; A. van ARSDALL et alii, "The Mandrake Plant...", op. cit., pp. 319-321. Amato Lusitano se refiere a ello en su comentario (línea 20; cf. n. 25). Basándose precisamente en tal creencia popular compuso Maquiavelo en 1518 su célebre comedia de enredo La mandragola: cf. D. WitTLin, Mandragora..., op. cit., pp. 247-249.

4 También se alude al asunto en el texto de Amato (líneas 26-36), aunque los precedentes son numerosos, como se dirá en el lugar referido (cf. n. 28). Para la falsificación de la mandrágora, véase D. WITTLIN, Mandragora..., op. cit., pp. 259-263.

5 Cf. Maria Patera, "Les rites d'extraction des plantes dans l'Antiquité. Magie, botanique et religion. L'exemple de la mandragore", Revue des Archéologues et Historiens d'Art 27 (1994), pp. 21-34; D. WITTLIN, Mandragora..., op. cit., pp. 238-240; A. van ARSDALl et alii, "The Mandrake Plant...", op. cit., pp. 295-319. Amato, como se verá (líneas 32-33), asocia con la falsificación de la mandrágora sus ritos de extracción.

6 ThPHR., HP, 9.8.8. La cita se repite en PLiN., Nat., 25.147.

7 I., BI, 7.25. La escena se narra aún con más detalle en el Herbarius de PSEUdo ApuLEYo, un texto farmacológico y botánico de los siglos IV-V: allí, por evitar sacrificar a un perro, se recomienda también el uso de una pértiga (Ps. Apul., Herb., 131). En la Edad Media, por lo demás, se añadió el tópico de los alaridos: la planta gritaría tanto al ser arrancada que el recolector debería ir siempre con los oídos tapados. Andando el tiempo, y sobre todo ya en la Edad Media y el Renacimiento, se añadieron algunas otras fábulas como, por ejemplo, la historia — con el fondo a la vista del esperma primigeniode que la mandrágora nacía del semen y la orina de los ahorcados y otros ajusticiados por tortura, de especial arraigo en zona germana: cf. D. WITTLIN, Mandragora..., op. cit., pp. 240-242; A. van ARSDALL et alii, "The Mandrake Plant...", op. cit., pp. 330-333. 
descripciones morfológicas más o menos ajustadas y en donde se destacaron sus cualidades medicinales más activas. En la Historia de las plantas, por ejemplo, enumera Teofrasto algunos empleos médicos de la mandrágora, que luego se recogerían en Dioscórides: las hojas con miel se aplican para curar heridas; la raíz majada con vinagre vale contra erisipelas, gota e insomnio; y se usa como ingrediente de filtros amorosos ${ }^{8}$. No obstante, Teofrasto describe también la mandrágora en otro lugar como una planta de frutos negros, semejantes a granos de uva y de sabor vinoso. La contradicción de esta especie con la mandrágora tradicional, tiempo después descrita por Dioscórides, llevó ya a algunos farmacólogos renacentistas a ofrecer para ella una identificación diferente y a relacionarla más concretamente con la belladona (Atropa belladona L.), tal como hoy día se admite?

No obstante, el texto científico de la Antigüedad que mejor describe la planta y sus principales usos médicos, ya sin apenas referencias mágicas ni populares, es la obra botánica de

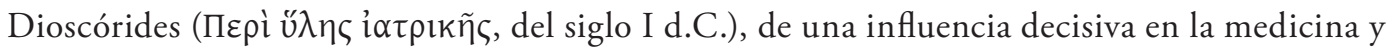
la farmacología desde la Antigüedad al Renacimiento ${ }^{10}$. Dioscórides distingue, de partida, dos especies. A la primera la llama hembra y negra, también conocida por $\theta \rho 1 \delta \alpha$ kí $\alpha$, sin tallo, de hojas parecidas a las de la lechuga, aunque más pequeñas, malolientes, extendidas por tierra, con frutos en forma de manzanas de color amarillento, olorosas, en las que hay unas semillas semejantes a las de la pera, con una raíz doble o triple, grande, negruzca, negra por fuera y blanca por dentro. La otra especie es la blanca y macho, a la que también llaman $\mu \omega ́$ prov (“enloquecedora”), de hojas blancas, grandes, anchas, lisas, semejantes a las de la acelga, con frutos mayores que los de la otra especie, de color azafrán, de olor pesado, que suelen comer los pastores y se quedan adormecidos, con raíz semejante a la anterior y también sin tallo. Los estudiosos suelen identificar ambas especies, respectivamente, con la Mandragora autumnalis L. y la Mandragora officinarum L. (= Mandragora vernalis Bertol.; Atropa mandragora L.), las dos especies hoy reconocidas como propias de la zona mediterránea, que junto a la de zona

8 THPHR., HP, 9.9.1. Una valoración del pasaje puede verse en la edición de Suzanne AmiGUES en THÉOPHRASTE, Recherche sur les plantes. Paris, Les Belles Lettres, 2003, pp. 130-131. No obstante, ya en el Corpus Hippocraticum (Fist., 890; Loc. Hom., 39; Mul., 1.621; Morb., 3.2.474) se citaba también la mandrágora como remedio casi siempre sedante y calmante, aplicado a veces a enfermos mentales.

9 ThPhr., HP, 6.2.9. En la traducción de S. AMIGUES, por ejemplo, se emplea directamente el término "belladone" para esta mandrágora (THÉOPHRASTE, Recherche..., op. cit., pp. 35 y 138).

10 Como estudios básicos sobre Dioscórides baste ver John M. RIDDLE, "Dioscorides", in F. Edward KRANZPaul O. KRISTELLER (eds.), Catalogus translationum et commentariorum. Mediaeval and Renaissance Latin Translations and Commentaries, vol. 4. Washington D.C., Catholic University of American Press, 1980, pp. 1-143; John M. RIDDLE, Dioscorides on Pharmacy and Medicine. Austin, University of Texas Press, 1985; Alain TouwAIDE, "Le Traité de matière médicale de Dioscoride. Pour une nouvelle lecture", Bulletin du Cercle Benelux d'Histoire de la Pharmacie 78 (1990), pp. 32-39; Alain TouWAIDE, "La Thérapeutique médicamenteuse de Dioscoride à Galien: du pharmaco-centrisme au médico-centrisme", in Armelle Debru (ed.), Galen on Pharmacology, Philosophy, History and Medicine. Leiden, Brill, 1997, pp. $255-282$. 
himalaya (M. caulescens Clarke) y tierras turcas (M. turcomanica Mizg) constituirían el grueso de las especies que integran el género Mandragorae ${ }^{11}$.

El resto del capítulo lo dedica Dioscórides a usos médicos: ofrece formas para obtener su zumo y enseña a hacer una cocción con vino que sirve de somnífero, calmante y sobre todo anestésico, pero también de purgante. En forma externa y en varios preparados tiene poder oftálmico y analgésico, vale para expulsar fetos muertos y menstruación retenida, resuelve durezas, lamparones, apostemas y diviesos, y sana la erisipela. A veces puede emplearse como antídoto de mordeduras de serpiente. El fruto purga la matriz y corta la hemorragia menstrual. Algunos de los usos médicos destacados, en efecto, obedecen a la composición propia de la mandrágora: como casi todas las solanáceas, posee cierta riqueza en alcaloides, en especial hiosciamina, atropina, hioscina y escopolamina - que comparte con otras especies como el beleño o la belladona - lo que hace de ella una planta alucinógena, narcótica e hipnótica, si bien hoy día - a diferencia de la belladona misma - no se usa en farmacología científica. Aun así, en homeopatía se le siguen reconociendo algunas cualidades ya vigentes en la Antigüedad: sedante, anti-infecciosa, activa contra la debilidad de la vejiga o laxante ${ }^{12}$.

Al final del capítulo, por lo demás, añade Dioscórides breve información, que parece citar de oídas, sobre una tercera y enigmática especie de mandrágora, a la que también llamarían $\mu \omega ́$ prov. Se trataría de una variedad parecida a la blanca y macho, solo que más tóxica y virulenta en sus efectos paralizantes y anestésicos. A tenor de la imprecisa descripción dioscorídea, la identificación moderna resulta difícil, aunque tiende a considerarse una mera variante de la Mandragora officinarum L., a la que Bertoloni llamó Mandragora vernalis por oposición a la autumnalis.

Tanto las dos especies primeras como la enigmática tercera variedad de mandrágora se incorporaron a la tradición botánica y farmacológica posterior como elementos casi puramente librescos, sin apenas aportaciones nuevas a los datos de Dioscórides, más allá de una rica y amplia representación iconográfica ${ }^{13}$. Para el caso de la tercera especie, además, ni en la Antigüedad misma ni en la Edad Media árabe o latina se hicieron elucubraciones sobre su identificación. Llegado el Renacimiento, fue Ermolao Barbaro (1454-1493) quien propuso una posible relación del morion con la berenjena (Solanum melongena L.), luego rechazada por casi todos los autores, incluido Amato Lusitano, aun cuando los botánicos de zona germana

11 Dsc. 4.75. Para la identificación y descripción científica baste ver D. Wittlin, Mandragora..., op. cit., pp. 10-29; A. van Arsdall et alii, "The Mandrake Plant...", op. cit., pp. 289-292; Christophe Maclaren, Quelle est la part de réalité dans le mythe de la mandragore?, publicado en 2009 y accesible en Inlibroveritas.net (http://www.inlibroveritas.net/lire/oeuvre26291.html), pp. 7-11.

12 Véase D. Wittuln, Mandragora..., op. cit., pp. 48-82, 198-272, 200-252; V. HAMBEL, Die alte Heydnische..., op. cit., pp. 13-17; C. MACLAREN, Quelle est la part..., op. cit., pp. 13-16.

13 Una visión de conjunto sobre ello puede verse en Minta Collins, Medieval Herbals. The Illustrative Tradition. Toronto, University of Toronto Press, 2000, pp. 31-114. 
(en especial Bock [1498-1554], Cordus [1515-1544] y Fuchs [1501-1566]) tendieron a identificarla con la segunda mandrágora de Teofrasto, es decir, con la belladona ${ }^{14}$.

\section{EL COMENTARIO DE AMATO}

Desde finales del siglo XV y a lo largo de todo el XVI el De materia medica de Dioscórides fue objeto de numerosas ediciones griegas y traducciones latinas y vernáculas a menudo acompañadas de comentarios filológicos, botánicos y farmacológicos ${ }^{15}$. Uno de ellos fue el de Amato Lusitano: tras un comentario parcial de juventud titulado Index Dioscoridis (Amberes 1536), publicó en Venecia en 1553 un comentario latino completo (las Enarrationes) a los cinco libros del botánico griego, sin inclusión del texto original. Es aquí, pues, donde se encuentra el breve comentario a la mandrágora objeto de este trabajo, cuya estructura se desarrolla como sigue.

1. Nomenclatura. Como hace en toda la obra y según era frecuente en casi todas las traducciones y comentarios a Dioscórides del Renacimiento, Amato pone antes del texto una serie de nombres con que se designaba la mandrágora en diferentes lenguas, tanto antiguas (griego y latín) como modernas (lenguas hispánicas, italiano, francés y alemán). Algunos de los nombres latinos procedían ya de la tradición genuina de Dioscórides, aunque otros se añadieron en la recensión interpolada, base de las primeras ediciones del Renacimiento ${ }^{16}$.

14 Usamos para las fuentes las abreviaturas resueltas al final de esta introducción: BARBARo, Cor., 79v; Bock, Stirp., 304-305; Cordus, Ann., 509-510; Fuchs, Hist., 529 y 532. La identificación de morion y belladona, no obstante, fue rechazada por autores como MATTIOLI, Disc., 597. Ya casi al final del siglo XVI propuso CesalPINo (Plant., 216) su identidad con el jazmín (Iasminum officinale L.). Sobre ello volvemos en las notas 29 y 30.

15 Para la difusión de Dioscórides en el Renacimiento pueden verse, por ejemplo, Jerry STANNARD, "Dioscorides and Renaissance Materia Medica", in Markus FlorkIN (ed.), Analecta Medico-Historica. I. Materia Medica in the XVIth Century. Oxford, Pergamon, 1966, pp. 1-21; J. M. RIDDLE, "Dioscorides" op. cit.; Alain TouWAIDE, Le Traité de matière médicale de Dioscoride à la Renaissance. Bruxelles, Université Catholique de Louvain, 1984. Para la botánica y la farmacología renacentistas son interesantes Agnes R. ARBER, Herbals. Their Origin and Evolution. A Chapter in the History of Botany, 1470-1670, W.T. STEARN (ed.). Cambridge, Cambridge University Press, 19863; Peter DILG, "Die botanische Kommentarliteratur in Italien um 1500 und ihr Einfluss auf Deutschland", in August Buck-Otto HERDInG (eds.), Der Kommentar in der Renaissance. Boppard, H. Boldt Verlag, 1975, pp. 225-253; Karen Meier ReEDS, Botany in Medieval and Renaissance Universities. New York-London, Garland Publishing, 1991; Paula FInDLEN, Possessing Nature: Museums, Collecting and Scientific Culture in Early Modern Italy. Berkeley-London, University of California Press, 1994; Brian W. OGILVIE, The Science of Describing: Natural History in Renaissance Europe. Chicago, University of Chicago Press, 2006.

16 Un buen ejemplo de dicha recensión, que combina la tradición genuina y la alfabética, puede ser el códice de Salamanca 2659, f. 121r: cf. Alain TouwAIDE, "The Salamanca Dioscorides (Salamanca, University Library, 2659)", Erytheia 24 (2003), pp. 125-158; Antonio LóPEz EIRE-Francisco CoRTÉs GabAUDÁn, Dioscórides, Sobre los remedios medicinales. Manuscrito de Salamanca: estudios y traducción. Salamanca, Universidad de Salamanca, 2006, p. 322. Para la etimología y los nombres de la planta, también en las lenguas modernas, puede verse D. WITTLIN, Mandragora..., op. cit., pp. 30-32 y 33-46; V. HAMBEL, Die alte Heydnische..., op. cit., pp. 19-21. Véase además la n. 19. 
2. Experiencia propia. Amato empieza su comentario reconociendo que nunca había visto la mandrágora en tierras hispanas, aun cuando era planta muy frecuente en Italia y, en especial, en Ferrara, donde había vivido entre 1540 y $1547^{17}$.

3. Descripción botánica. Sin entrar a decir si se refiere a la especie macho o a la hembra, Amato describe la planta que se ve habitualmente en los huertos de Italia. Hace unas primeras alusiones eruditas a Pitágoras y Columela, probablemente de segunda mano.

4. Recomendación medicinal de Dioscórides: vino de mandrágora.

5. Falsificación. Dedica una buena cantidad de texto a denunciar, como era ya tópico entre los farmacólogos del Renacimiento, la falsificación de la mandrágora entre charlatanes y curanderos, que se aprovechaban de sus supuestas virtudes contra la esterilidad femenina para venderla a precios muy elevados. En su denuncia, describe el proceso de falsificación y las creencias sobre su condición antropomórfica y sus especiales ritos de extracción. Termina reclamando la prohibición en Italia de dichas ventas fraudulentas, tal como - afirma - se hacía ya en tierras hispanas, en posible referencia al Tribunal del Protomedicato ${ }^{18}$.

6. Tercera especie. La parte final del texto se reserva para comentar de pasada la posible asociación del morion de Dioscórides con la berenjena. Según habían hecho ya otros comentaristas, rechaza Amato la identificación y aprovecha para aludir a las cualidades alimenticias de las berenjenas, ya por entonces consumidas habitualmente en Italia. Pese a todo, considera

17 En 1533, y a tenor de los problemas que cada vez más acuciaban a los judíos conversos en Portugal y España, Amato emigró a Amberes para trabajar en el negocio de las especias que allí dirigía, entre otros, Henrique Pires, tío carnal suyo. En Amberes, como comerciante y médico, vivió hasta finales de los años treinta. En 1540, siguiendo a su propia familia, se estableció en Ferrara, donde vivió hasta 1547, año en que pasó a Ancona hasta su huida de 1555 y su paso posterior a Dubrovnik y Salónica. En Italia adquirió gran reputación como médico práctico y allí publicó las Enarrationes y sus primeras Centuriae medicinales. Véase, con bibliografía reciente, António M. Lopes ANDRADE, "A Senhora e os destinos da Nação Portuguesa: o caminho de Amato Lusitano e de Duarte Gomes", Cadernos de Estudos Sefarditas 10-11 (2011), pp. 87-130; António M. Lopes ANDRADE, "De Antuérpia a Ferrara: o caminho de Amato Lusitano e da sua família", Medicina na Beira Interior. Da Pré-História ao séc. XXI - Cadernos de Cultura 25 (2011), pp. 5-16.

18 Fundado en 1471 por los Reyes Católicos (en principio solo para la Corona de Castilla, pero pronto extendido a todo el reino y más tarde a las colonias americanas) y vigente hasta el siglo XIX, el Real Tribunal del Protomedicato se encargaba de controlar el ejercicio de la medicina y la farmacia, examinar y dar licencia a médicos y boticarios y controlar el intrusismo profesional. Sobre el asunto pueden verse los artículos recogidos en un dossier especial de la revista Dynamis 16 (1996), pp. 17-259; un estudio detallado es el de Ma Soledad Campos Díez, El Real Tribunal del Protomedicato castellano. Siglos XIV-XIX. Cuenca, Universidad de Castilla La Mancha, 1999; una síntesis de conjunto está también en Juan Riera Palmero, Protomedicato, humanismo y medicina en Castilla. Valladolid, Universidad de Valladolid, 2000. Por lo demás, otros países tuvieron también una institución parecida, como fueron los casos de Portugal o el reino aragonés de Nápoles: véase David GeNTILCORE, "All That Pertains to Medicine: Protomedici and Protomedicati in Early Modern Italy", Medical History 38 (1994), pp. 121-142. A ello, asimismo, debe sumarse el control específico ejercido por las cofradías de médicos, cirujanos y boticarios y, sobre todo, la amenaza omnipresente de la Inquisición, en especial cuando el asunto tocaba a superstición y hechicería. 
la existencia de una especie de berenjenas de frutos amarillentos, para la que admite en cambio una posible identidad con la tercera mandrágora de Dioscórides.

7. Cita galénica. Termina el comentario con una cita de Galeno respecto a las virtudes de la mandrágora.

\section{EDICIÓN LATINA}

Para el texto latino del comentario a la mandrágora de Amato hemos colacionado todas las ediciones existentes y hemos mantenido el siguiente criterio: el texto base es la edición princeps de Venecia de 1553, aunque sus errores y posibles malas lecturas se corrigen con la segunda edición de Estrasburgo de 1554 y la cuarta de Lión de 1558, cuidada por Robert Constantin y única que incluye ilustraciones procedentes de las obras de Leonhart Fuchs y Jacques Daléchamps. La edición de Lión se realizó en cuatro tiradas diferentes, que salieron en imprentas distintas (Roville, Payen, viuda de Arnouillet y Bonhomme) y que son exactamente iguales, por lo que nos hemos servido solo de la primera de ellas para la colación. Se desecha, en fin, la tercera edición de Venecia de 1557 por ser un codex descriptus de la princeps. A los textos manejados les hemos nombrado de la siguiente manera:

\footnotetext{
$\mathrm{V}=$ In Dioscoridis Anazarbei De materia medica libros quinque enarrationes eruditissimae doctoris Amati Lusitani medici ac philosophi celeberrimi..., Venetiis, apud Gualterum Scotum, 1553, pp. 413-414.
}

$\mathrm{A}=$ In Dioscoridis Anazarbei De materia medica libros quinque enarrationes eruditissimae doctoris Amati Lusitani medici ac philosophi celeberrimi..., Argentorati excudebat VVendelinus Rihelius, 1554, pp. 431-432.

$\mathrm{L}=$ In Dioscoridis Anazarbei De materia medica libros quinque enarrationes eruditissimae doctoris Amati Lusitani medici ac philosophi celeberrimi..., Lugduni, apud Gulielmum Rovillium, sub scuto Veneto, 1558, pp. 647-649.

El texto latino respeta la notación original y propia de las ediciones renacentistas, excepto en algunos casos concretos, como la distinción entre $u$ y $v$, que se mantiene igual que en romance. Se resuelven todas las abreviaturas. Las citas literales se ponen entre comillas y las citas de títulos de obras van siempre en cursiva. El texto latino respeta la disposición del original: título, entradilla con sinónimos y comentario. Aunque el texto del comentario va en origen 
todo seguido, se ha preferido disponerlo aquí en párrafos y puntuarlo conforme a las reglas habituales del español moderno.

\section{TRADUCCIÓN Y ANOTACIÓN}

La traducción castellana, primera de este texto, trata de ser fiel en lo posible al original latino, aunque guardando siempre el decoro necesario en la lengua de llegada. Debido a las peculiaridades propias de todo texto técnico, se ha incluido una anotación más o menos amplia que dé cuenta de todos los aspectos relevantes del original: aclaración de términos oscuros, identificación de plantas, aspectos de la vida y la obra del autor, detección de fuentes expresas y otras no citadas, etc.

Respecto a las fuentes, se ha considerado imprescindible rastrear los comentarios a Dioscórides previos al de Amato, así como otros textos botánicos más generales, pues el médico portugués los tuvo muy en cuenta y en algunos casos constituyeron la base de su obra, en especial los textos de Ermolao Barbaro, Marcello Virgilio, Antonio Musa Brasavola y, sobre todo, Pietro Andrea Mattioli. A ellos y otros autores más se remite cada vez que se encuentra una posible deuda o paralelo, sin desdeñar tampoco otras obras posteriores que puedan aportar alguna noticia significativa para entender mejor el comentario de Amato.

\section{ABREVIATURAS}

Por evitar repeticiones prolijas, consideramos conveniente citar de forma abreviada todas las fuentes a que se remite en las notas a la traducción del texto. Se ofrece aquí, por tanto, la equivalencia de tales abreviaturas, con breves comentarios — cuando así se requiera- sobre su fecha de composición.

Avicena, Canon = Avicennae Liber canonis, Venetiis, Iunta, 1527.

Barbaro, Diosc. $=$ Pedacii Dioscoridis Anazarbei de medicinali materia ab eodem Barbaro Latinitate primum donati libri quinque, Venetiis, Barbari, 1516. Edición póstuma de la traducción latina de Ermolao Barbaro (posiblemente de los ańos 1481/82), a cargo de Giovanni Battista Cipelli (Egnatius).

Barbaro, Cor. = Hermolai Barbari ... Corollarium, Venetiis, Barbari, 1516. En la misma edición anterior se incluyen unos comentarios de Barbaro, redactados posiblemente en 1487.

Bock, Stirp. = Heronymi Tragi de stirpium ... usitatis nomenclaturis, propriisque differentiis, neque non temperaturis ac facultatibus commentariorum libri tres, Argentorati, Rihelius, 
1552. Se trata de la versión latina de David Kendel de la obra que Bock había publicado originariamente en alemán: Neu Kreutterbuch, Frankfurt 1539.

Brasavola, Ex. = Antonii Musae Brassavolae Ferrariensis Examen omnium simplicium medicamentorum, quorum in officinis usus est, Lugduni, Frellonii, 1537.

Cesalpino, Plant. = De plantis libri XV Andreae Cesalpini, Florentiae, Marescottus, 1583.

Cordus, Ann. $=$ Valerii Cordi Simesusii Annotationes doctissimae in Dioscoridis de medica materia libros, Argentorati, Rihelius, 1549. Es una edición póstuma de las anotaciones de Cordus (datadas entre 1539 y 1543) efectuada por Walther Ryff. Más adelante las reeditaría Conrad Gesner (Argentorati, Rihelius, 1561).

Fuchs, Hist. = De historia stirpium commentarii insignes ... Leonharto Fuchsio ... autore, Basileae, Isingrinus, 1542.

Galeno, Simpl. = De simplicium medicamentorum temperamentis ac facultatibus, en K. G. Kühn, ed., Claudi Galeni opera omnia, vol. 7, Leipzig, Cnobloch, 1824.

Laguna, Diosc. $=$ Pedazio Dioscorides Anazarbeo, Acerca de la materia medicinaly de los venenos mortiferos, Amberes, Laet, 1555.

Mattioli, Disc. = Il Dioscoride dell'eccelente dottore medico P. Matthioli da Siena con le suoi discorsi..., Venezia, Valgrisi, 1550. Citamos por esta edición de la versión italiana y los comentarios de Mattioli (princeps de Venecia 1544), que correría luego paralela a la edición latina (princeps de Venecia 1554).

Ruelle, Diosc. $=$ Pedacii Dioscoridis Anazarbei de medicinali materia libri quinque ... Ioanni Ruellio Suesionensi interprete, Parisiis, Henricus Stephanus, 1516.

Virgilio, Diosc. $=$ Pedacii Dioscoridae Anazarbei de medica materia libri sex, interprete Marcello Virgilio, Venetiis, Iunta, 1518.

Virgilio, Ann. = Pedacii Dioscoridae Anazarbei de medica materia libri sex, interprete Marcello Virgilio..., cum eiusdem annotationibus, Venetiis, Iunta, 1518. A la traducción anterior añadió Marcello Virgilio unos comentarios. 


\section{Texto Latino}

\section{DE MANDRAGORA}

Graece $\mu \alpha v \delta \rho \alpha \gamma o ́ \rho \alpha \varsigma$. Latine mandragora, pomum terrestre, pomum caninum, Circe. Hispanice mandracola yerva. Italice la mandragora. Gallice mandragore.

Germanice Alraun.

Enarratio 79

Nunquam quod equidem meminerim in Hispania mandragoram vidi. In Italia vero frequens est herba, praecipue Ferrariae, ubi nullus fere extat hortulus in quo mandragora non reperiatur. In coenobio enim Carthusianorum, apud quod unusquisque monachus suum proprium et particularem hortulum habet, ita felicissime crescit, ut nullus extet qui non hac decoretur herba.

Crescit autem folio lapathi, lato, oblongo, per terram strato, ad cuius radices poma quinque vel sex numero nascuntur, magnitudine parvae pilae, coloris interdum virescunt herbacei; quum vero ad maturitatem perveniunt, lutei saturi efficiuntur. Nascuntur autem, ut obiter hoc attingamus, poma haec pediculis semi dodrantalis altitudinis ita apposite, ut unumquodque pomorum homuncionem quendam absque brachiis repraesentare videatur, qua de causa Pythagoras mandragoram 'anthropomorphon', id est, figura hominis, appellavit, quem secutus Columella mandragoram in suo Horto 'semihominem' dixit. Cui signatae causae alteram addimus vulgarem: quia mandragora radicem in duas fibras, veluti duo crura, divisam habet, unde quoque 'semihomo' appellatur. Haec etiam 'Circe' dicebatur, quia ea ad amatoria et veneficia Circe famosissima incantatrix utebatur. Et de ea in Pentateucho legitur, quam Hebraei 'dudaim' sua lingua vocant.

Ex huius vero radice vinum mandragorite parandum Dioscorides et medici suadent, quod plerunque propinant iis quibus secare aut membrum aliquod urere volunt: mirifice enim stupefacit.

Iustum erit hic enarrare tyrannidem ac imposturam nugivendulorum ac impostorum hominum, qui homunculos quosdam, ex radicibus cannarum sculptos, mulieribus

3 yerua VA: om. $L$ || la VA: om. $L$ || post mandragora add. Arabice tabora $L$ || post mandragore add. et mandegloire $L \cdot \mathbf{5} 79 \mathrm{VA}$ : $L X X I X L \cdot \mathbf{1 5}$ homuncionem $A L$ : homuntionem $V \cdot \mathbf{2 5}$ mirifice $A L$ : mirificae V. 
sterilibus et non parturientibus loco mandragorae non parvo vendunt pretio. Sculpunt enim hominis effigiem in cannarum, ut dixi, radice ita apposite et solerter ut, ubi opus est appareant capilli, ut in barba, capite et pectine, grana tritici quaedam affingant quae, cum radice simul terra obruta, plures, tenues ac capillis similes radices emittunt, ac inde tota ipsa radix figura homuncionem repraesentat, quam e terra non nisi obturatis auribus eradicari posse vel canis caudae adalligatam alta mente nugantur. Vendunt autem unam vel alteram harum radicem triginta, nonnunquam vero quadraginta aureis ducatis. 35 Pereant igitur balatrones ii falsarii, quos ut Italia universa fert, ita tota Hispania eos fugat et non permittit imperitum vulgus imposturis circumveniri.

Tertia vero species mandragorae 'morion' dicitur, quae procul dubio melongena non est, ut nonnulli opinati sunt. Est enim melongena vel melezana fructus ubique familiaris, quem aliqui 'berengenam' vel 'belongenam', alii vero 'Cupidinis pomum' 40 vel 'insanum pomum' appellant, quod inter cucurbitas et melones seritur, et victui non aspernendum obsonium esset, nisi tristes ac atrabiliarios gigneret humores, ut Avicennam adnotasse legimus. Huius porro altera species in Italia cernitur, pomum gignens luteum, quam ego morion esse dixissem, quia cum mandragora magnam cum figura tum viribus habet similitudinem, de quibus Galenus libro septimo De facultatibus simplicium medicamentorum ita tradit: "Refrigerat tertio excessu mandragora, nonnihil tamen pomis caloris atque humiditatis inest, unde soporem conciliant. Radicis cortex non tantum refrigerat, sed etiam siccat. Quod eo includitur imbecillimum est".

32 homuncionem $A L$ : homuntionem $V \cdot \mathbf{4 6}$ nonnihil $A L$ : uon nihil $V$. 


\section{Traducción y Anotación}

\section{MANDRÁGORA}

Griego: $\mu \alpha v \delta \rho \alpha \gamma o ́ \rho \alpha c$. Latín: mandragora, pomum terrestre, pomum caninum, Circe. Español: mandracola yerva. Italiano: la mandragora. Francés: mandragore.

Alemán: Alraun ${ }^{19}$.

Nunca, que yo recuerde, vi la mandrágora en tierras hispanas ${ }^{20}$. En Italia, sin embargo, es una hierba frecuente, sobre todo en Ferrara, donde casi no hay huertecillo en que no se encuentre la mandrágora. En el cenobio de los cartujos, de hecho, donde cada monje tiene su propio y particular huertecillo, crece de forma tan abundante que ninguno hay sin el adorno de tal hierba ${ }^{21}$.

Crece con hojas como las de la romaza, anchas, largas, echadas por tierra, junto a cuyas raíces nacen cinco o seis manzanas del tamaño de una pelota pequeña, de color herbáceo cuando están en sazón, aunque cuando maduran se vuelven de un tono amarillo oscuro ${ }^{22}$. Nacen tales manzanas, por tocar el asunto de paso, en pedículos de cuatro pulgadas y media

15 de longitud, y en una disposición tal que cada manzana parece representar un hombrecillo sin brazos, de ahí que Pitágoras la llamase antropomorphon, es decir, "con figura humana”, a

19 Las expresiones pomum terrestre y pomum caninum remontan a los mala terrestria y mala canina documentados en las tradiciones alfabética e interpolada de Dioscórides, que incorporan una serie de sinónimos de plantas en numerosas lenguas (los llamados notha). Por otro lado, cuando Amato dice Hispanice puede aludir a términos tanto españoles como portugueses: en este caso, "mandrácola" es un hispanismo que se sigue usando en ciertas partes de España, como también la expresión "uva de moro" (el DRAE, no obstante, solo trae "mandrágora" y "mandrágula", nombre citado en LAGUNA, DioSC., 424). Por lo demás, en la edición lionesa de 1558, y posiblemente por obra de Constantin, se añade el sinónimo "mandegloire", usado en francés antiguo, así como el arabismo tabora (erróneamente notado por iabora).

20 Como antes con Hispanice, cuando Amato cita Hispania puede estar refiriéndose a cualquier zona de la Península Ibérica, bien fuese España o Portugal. En cualquier caso, y pese al testimonio de Amato, la mandrágora no es infrecuente de tales zonas (nace silvestre sobre todo en tierras andaluzas).

21 Amato se refiere al monasterio de la Cartuja de Ferrara, fundado en 1452 y desde el siglo XIX cementerio monumental. Hay en MATTIOL noticias similares sobre la abundancia de la planta en Italia, con las que comienza su comentario al capítulo (Disc., 600), para el que parece tomar como base a Brasavola, Ex., 312. Contrasta el dato con la escasez de la planta en tierras alemanas, como se lee en Bock, Stirp., 891, quien reconoce no haberla visto nunca.

22 La comparación de las hojas de mandrágora (sin especificar aquí la especie) con las de la romaza (Rumex obtusifolius L.) se lee ya en Plinio, Nat., 25.147, lo que recoge BARBARO, Cor., 79v. Del tamaño y el color del fruto habla en extenso, entre otros, VIRGILIO, Ann., $247 \mathrm{r}$. 
quien siguió Columela, que en su Huerto designó a la mandrágora 'semihombre'. A la causa aducida podemos añadir otra más conocida: que la mandrágora tiene la raíz bifurcada en dos lóbulos, como si fuesen dos piernas, de ahí que también se la llame 'semihombre'23. Asimismo, se le decía 'Circe', porque la famosísima hechicera Circe usaba de ella para sus filtros amorosos y mágicos ${ }^{24}$. Y de ella se habla en el Pentateuco: los hebreos en su lengua la llaman dudaim ${ }^{25}$.

Dioscórides y los médicos recomiendan que con su raíz se prepare un vino de mandrágora que la mayoría administra a quienes van a sajar o quemar algún miembro, pues anestesia admirablemente ${ }^{26}$.

Justo será narrar aquí la tiranía e impostura de vendedores de baratijas ${ }^{27}$ e impostores que a no escaso precio, y como si fuese mandrágora, venden a mujeres estériles y que no se embarazan unos hombritos esculpidos en raíces de cañas. Como digo, en la raíz de las cañas esculpen la figura de un hombre con tan buena disposición y habilidad que, donde se precisa que nazca vello - en la barba, la cabeza y el pubis - incrustan unos cuantos granos de trigo que, al enterrarse con la raíz, producen a su vez unas raicillas delgadas y semejantes a pelos, y después la raíz entera adquiere la forma de un hombrecillo, que con grandilocuencia fingen no puede arrancarse de tierra sin los oídos tapados, o bien atándola al rabo de un perro. Y venden una o dos raíces de estas por treinta y a veces hasta por cuarenta ducados de oro. Ojalá perezcan esos

23 La referencia al anthropomorphos pitagórico está ya en los antiguos Notha de la tradición interpolada. Aluden a ello algunos comentaristas de Dioscórides y otros botánicos del Renacimiento, no sin censurar que se haya querido establecer una relación literal entre planta y hombre: BARBARO, Cor., 79v; VIRGILIO, Ann., 247r; Bock, Stirp., 891; MATTIOLI, Disc., p. 601. Pero Amato, de forma original, establece una doble causa: primero considera que la relación planta / hombre procedería del fruto, semejante a una figura humana sin brazos, y luego remite a la explicación más vulgar y común: el parecido de la raíz con dos piernas humanas. Cita, asimismo, el semihomo de Columela (ColvM., 10,19-20), en referencia que sigue de cerca a MATTIOLI, Disc., 601 (a su vez dependiente de BrASAVOLA, Ex., 313), quien arremete sin nombrarlo contra VIRGILIO (Ann., 247r) por no haber comprendido la metáfora y haber censurado a los dos clásicos.

24 El término viene ya en la tradición genuina de Dioscórides, solo que en forma adjetiva: Kipkaía. La relación directa con Circe está también en la tradición interpolada, y con más o menos amplitud se recoge en algunas traducciones latinas renacentistas, como la de BARBARo, Diosc., 83v, y sobre todo la de VIRGILIO, DiosC., 246v, fuente directa aquí de Amato: qui Circeam vocarunt nomen illi a Circe fecerunt, quoniam creditur radix eius amatoriis veneficiis utilis esse.

25 Así en el Génesis (Gen. 30.24), donde se alude a la planta por sus bondades para la fertilidad, en este caso de Raquel, que con su ayuda logró concebir a José (también se cita, en otro contexto, en el Cantar de los cantares: Cant. 7.13). Bock, Stirp., 891-893, hace un amplio excursus sobre la mandrágora en fuentes hebreas. Cf. Introducción, n. 2.

26 El vino de mandrágora (hecho de los frutos, la corteza de la raíz o la raíz entera) es una receta que trae Dioscórides en el capítulo propio dedicado a la planta (4.75) y que, al parecer, se empleaba desde tiempos antiguos como bebida útil para anestesiar. No obstante, vuelve a comentarlo con más detalle en 5.71. Amato usa para nombrarlo una transcripción del griego $\mu \alpha v \delta \rho \alpha \gamma o$ pírns, que ya se documenta en las primeras traducciones latinas de la obra: BARBARO, Diosc., 106V; RUELLE, DiosC., 125V; VIRGILIO, Diosc., 308. Un breve comentario a este vino está en BRASAVOLA, Ex., 312-313.

27 El término nugivendulus es un diminutivo del clásico nugivendus, ya atestiguado en PLAUTO (Aul., 525). 
35 tramposos charlatanes a quienes, si en Italia entera se los tolera, en todas las tierras hispanas se les persigue y no se permite que el pueblo sencillo se vea engañado con sus imposturas ${ }^{28}$.

La tercera especie de mandrágora se llama morion, y sin duda no es la berenjena, como algunos han creído ${ }^{29}$. Es, en efecto, la berenjena o "meleçana" un fruto muy común, que algunos llaman 'berengena' o 'belongena', otros 'manzana de Cupido' o 'manzana insana', que se siembra entre

40 las calabazas y los melones y que no sería un alimento despreciable en nuestra dieta si no generase humores tristes y atrabiliarios, como leemos que había dicho Avicena. En todo caso, una segunda variedad de ella puede verse en Italia, con manzanas amarillentas, que es la que yo identificaría con el morion, pues que presenta gran similitud con la mandrágora tanto en su forma como en sus virtudes medicinales ${ }^{30}$, de las que Galeno dice lo siguiente en el libro séptimo de Sobre las facultades de los medicamentos simples: "Enfría la mandrágora en grado tercero, aunque en sus manzanas tiene algo de calor y humedad, de ahí que provoquen sopor. La corteza de la raíz no solo enfría, sino que también seca. La parte de dentro es mucho más floja” ${ }^{31}$.

\section{REFERENCIAS BIBLIOGRÁFICAS}

Andrade, António M. Lopes, "A Senhora e os destinos da Nação Portuguesa: o caminho de Amato Lusitano e de Duarte Gomes", Cadernos de Estudos Sefarditas 10-11 (2011), pp. 87-130.

Andrade, António M. Lopes, "De Antuérpia a Ferrara: o caminho de Amato Lusitano e da sua família", Medicina na Beira Interior. Da Pré-História ao séc. XXI - Cadernos de Cultura 25 (2011), pp. 5-16.

28 La queja está ya en los principales autores que sirven de fuente habitual a Amato: VIRGILIO, Ann., 247r; Brasavola, Ex., 313; Bock, Stirp., 890-89; Cordus, Ann., 510a; Fuchs, Hist., 587; Mattioli, Disc., 600-601. Como se dijo en la Introducción (n. 7), los ritos de extracción aquí aludidos se narraban ya en Flavio Josefo y el Pseudo Apuleyo. Por lo demás, la última frase de Amato parece aludir al Tribunal del Protomedicato (cf. arriba, n. 18).

29 La tercera mandrágora de Dioscórides, hoy considerada una variante de la especie macho, recibió diferentes identificaciones en el Renacimiento, en especial berenjena, belladona y hasta jazmín (cf. arriba, n. 14). La asociación con la berenjena, luego desestimada por casi todos los autores, se debía a Barbaro, Cor., $79 \mathrm{v}$.

30 Como hacen otros comentaristas de Dioscórides (BARBARO, Cor., 79v; MATTIOLI, Disc., 601-602), aprovecha Amato la referencia a las berenjenas para aludir a algunos de sus nombres (casi todos medievales: cf. Mateo Silvático, Pandectae medicinae. Lugduni, T. Paganus, 1541, ff. 130ra-b) y recoger el tópico de su mala calidad alimenticia, para lo que cita a AvicenA (Canon, 2.455, s.v. Melongena), como ya había hecho el propio Mattioli. La planta, ausente de los herbarios antiguos, se describe en numerosas fuentes medievales y renacentistas: véase Marie Christine DaUnAY-Jules JANICK-Henri LATERROT, "Iconography of the Solanaceae from Antiquity to the 17th Century: A Rich Source of Information on Genetic Diversity and Uses", Acta Horticulturae 745 (2007), pp. 59-88. En todo caso, y pese a desestimar la identidad morion I berenjena, Amato habla de una especie de berenjena de fruto amarillo que, en su opinión, bien podría ser la tercera mandrágora de Dioscórides: su opción, en todo caso, no tuvo fortuna.

31 Véase Galeno, Simpl., 7.12.4 (KüHN 12.67). Amato cierra con la cita galénica a imitación de MATTIOLI, Disc., p. 602. 
Arber, Agnes R., Herbals. Their Origin and Evolution. A Chapter in the History of Botany, 1470-1670, W. T. Stearn (ed.). Cambridge, Cambridge University Press, $1986^{3}$.

Arsdall, Anne van; Klug, Helmut W.; Blanz, Paul, “The Mandrake Plant and its Legend: A New Perspective”, in Peter Bierbaumer \& Helmut W. Klug (eds.), Old Names-New Growth: Proceedings of the 2nd ASPNS Conference. Graz, Universität Graz, 2007, pp. 285-346.

Campos Díez, Ma Soledad, El Real Tribunal del Protomedicato castellano. Siglos XIV-XIX. Cuenca, Universidad de Castilla La Mancha, 1999.

Collins, Minta, Medieval Herbals. The Illustrative Tradition. Toronto, University of Toronto Press, 2000.

Daunay, Marie Christine; Janick, Jules; Laterrot, Henri, "Iconography of the Solanaceae from Antiquity to the 17th Century: A Rich Source of Information on Genetic Diversity and Uses", Acta Horticulturae 745 (2007), pp. 59-88.

Dilg, Peter, "Die botanische Kommentarliteratur in Italien um 1500 und ihr Einfluss auf Deutschland", in August Buck \& Otto Herding (eds.), Der Kommentar in der Renaissance. Boppart, H. Boldt Verlag, 1975, pp. 225-253.

Findlen, Paula, Possessing Nature: Museums, Collecting and Scientific Culture in Early Modern Italy. Berkeley -London, University of California Press, 1994.

Gentilcore, David, "All That Pertains to Medicine: Protomedici and Protomedicati in Early Modern Italy", Medical History 38 (1994), pp. 121-142.

Намвеl, Vera, Die alte Heydnische Abgöttische Fabel von der Alraun: Verwendung und Bedeutung der Alraune in Geschichte und Gegenwart. Diss., Passau, Universität Passau, 2002.

López Eire, Antonio; Cortés Gabaudán, Francisco, Dioscórides, Sobre los remedios medicinales. Manuscrito de Salamanca: estudios y traducción. Salamanca, Universidad de Salamanca, 2006.

Maclaren, Christophe, Quelle est la part de réalité dans le mythe de la mandragore?, 2009, accesible en Inlibroveritas.net (http://www.inlibroveritas.net/lire/oeuvre 26291.html).

Mateo Silvático, Pandectae medicinae. Lugduni, T. Paganus, 1541.

Ogilvie, Brian W., The Science of Describing: Natural History in Renaissance Europe. Chicago, University of Chicago Press, 2006.

Patera, Maria, "Les rites d'extraction des plantes dans l'Antiquité. Magie, botanique et religion. L'exemple de la mandragore", Revue des Archéologues et Historiens d'Art 27 (1994), pp. 21-34.

Reeds, Karen Meier, Botany in Medieval and Renaissance Universities. New York-London, Garland Publishing, 1991.

Riddle, John M., “Dioscorides”, in F. Edward Kranz \& Paul O. Kristeller (eds.), Catalogus translationum et commentariorum. Mediaeval and Renaissance Latin Translations and Commentaries, vol. 4. Washington D.C., Catholic University of American Press, 1980, pp. 1-143.

Riddle, John M., Dioscorides on Pharmacy and Medicine. Austin, University of Texas Press, 1985.

Riera Palmero, Juan, Protomedicato, humanismo y medicina en Castilla. Valladolid, Universidad de Valladolid, 2000. 
Roland, Jean-Denis, "La mandragore: le mythe d'une racine, la racine d'un mythe", Annales des Sciences Naturells, Botanique 2 (1990-1991), pp. 49-81.

Simoons, Frederik J., Plants of Life, Plants of Death. Madison-London, The University of Wisconsin Press, 1999.

Stannard, Jerry, "Dioscorides and Renaissance Materia Medica", in Markus Florkin (ed.), Analecta Medico-Historica. I. Materia Medica in the XVIth Century. Oxford, Pergamon, 1966, pp. 1-21.

Théophraste, Recherche sur les plantes, Suzanne Amigues (ed.). Paris, Les Belles Lettres, 2003.

Thomson, Charles J. S., The Mystic Mandrake. New York, University Books, 1968.

Touwaide, Alain, Le Traité de matière médicale de Dioscoride à la Renaissance. Bruxelles, Université Catholique de Louvain, 1984.

Touwaide, Alain, "Le Traité de matière médicale de Dioscoride. Pour une nouvelle lecture", Bulletin du Cercle Benelux d'Histoire de la Pharmacie 78 (1990), pp. 32-39

Touwaide, Alain, "La Thérapeutique médicamenteuse de Dioscoride à Galien: du pharmaco-centrisme au médico-centrisme", in Armelle Debru (ed.), Galen on Pharmacology, Philosophy, History and Medicine. Leiden, Brill, 1997, pp. 255-282.

Touwaide, Alain, “The Salamanca Dioscorides (Salamanca, University Library, 2659)”, Erytheia 24 (2003), pp. $125-158$.

Van Den Berg, Marlene; Dircksen, Marianne, "Mandrake from Antiquity to Harry Potter", Akroterion 53 (2008), pp. 67-79.

Wittunn, Dorit, Mandragora. Eine Arzneipflanze in Antike, Mittelalter und Neuzeit. Basel, Dietikon, 1999.

Zarcone, Thierry, “Le Mythe de la mandragore: la plante-homme”, Diogenes 207 (2004), pp. 140-173. 
A partir dos alvores do século XVI, a matéria médica torna-se indiscutivelmente um tema de primeira grandeza entre os membros da República das Letras, objecto de estudo e de controvérsia entre os mais notáveis humanistas europeus, em particular entre os cultores da arte médica. Entre os autores em destaque neste volume encontram-se, à cabeça, os nomes de Amato Lusitano, Garcia de Orta e Nicolás Monardes, famosos pelos contributos valiosos que deram para o conhecimento do mundo natural. 0 volume encontra-se dividido em duas partes: a primeira, subordinada ao título "Humanismo e Ciência", alberga os estudos que versam sobre todos os autores estudados, à excepção de Amato Lusitano; a segunda está reservada a um conjunto de trabalhos dedicados exclusivamente ao médico albicastrense, cuja autoria se fica a dever, em boa parte, aos membros da equipa do projecto de I\&D "Dioscórides e o Humanismo Português: os Comentários de Amato Lusitano", tomando, por isso, 0 seu próprio título. Nesta segunda parte, oferece-se, desde já, aos leitores uma amostra significativa do trabalho desenvolvido no âmbito do projecto e que culminará, assim se espera, na edição e tradução integral para língua portuguesa das quatro obras previstas de Dioscórides, Amato Lusitano e Pietro Andrea Mattioli. 
